\title{
Insect-specific virus evolution and potential effects on vector competence
}

\author{
Pontus Öhlund ${ }^{1} \cdot$ Hanna Lundén ${ }^{1} \cdot$ Anne-Lie Blomström ${ }^{1}[0$
}

Received: 1 October 2018 / Accepted: 21 December 2018 / Published online: 10 January 2019

(c) The Author(s) 2019

\begin{abstract}
The advancement in high-throughput sequencing technology and bioinformatics tools has spurred a new age of viral discovery. Arthropods is the largest group of animals and has shown to be a major reservoir of different viruses, including a group known as insect-specific viruses (ISVs). The majority of known ISVs have been isolated from mosquitoes and shown to belong to viral families associated with animal arbovirus pathogens, such as Flaviviridae, Togaviridae and Phenuiviridae. These insect-specific viruses have a strict tropism and are unable to replicate in vertebrate cells, these properties are interesting for many reasons. One is that these viruses could potentially be utilised as biocontrol agents using a similar strategy as for Wolbachia. Mosquitoes infected with the viral agent could have inferior vectorial capacity of arboviruses resulting in a decrease of circulating arboviruses of public health importance. Moreover, insect-specific viruses are thought to be ancestral to arboviruses and could be used to study the evolution of the switch from single-host to dual-host. In this review, we discuss new discoveries and hypothesis in the field of arboviruses and insect-specific viruses.
\end{abstract}

Keywords Insect-specific virus $\cdot$ Arbovirus $\cdot$ Vector competence $\cdot$ Evolution

\section{Introduction}

The first insect-specific virus (ISV) was discovered over 40 years ago by Stollar and Thomas [1]. It was isolated from an Aedes aegypti cell culture where a large number of syncytia were observed and the virus was named cell fusing agent virus (CFAV). Further, when inoculated on different vertebrate cell lines no cytopathic effect (CPE) could be observed and the virus could not be re-isolated, suggesting that the virus must be insect-specific [1]. Years after its discovery CFAV was molecularly characterised as a positive-sense RNA virus within the family Flaviviridae [2] and in 2006 it was isolated from field-caught mosquitoes in Puerto Rico [3]. Since the CFAV discovery a large number of ISVs have been discovered with increased

Edited by A. Lorena Passarelli.

Anne-Lie Blomström

anne-lie.blomstrom@slu.se

1 Section of Virology, Department of Biomedical Sciences and Veterinary Public Health, Swedish University of Agricultural Sciences, Box 7028, 75007 Uppsala, Sweden frequency in the last two decades due to the advancement in high-throughput sequencing, metagenomics and intensified mosquito surveillance [4]. Even though this group of viruses is called "insect-specific viruses" the majority have been discovered in mosquitoes and one can argue that for these viruses the term "mosquito-specific viruses" would be more appropriate. ISVs are restricted to arthropods and are unable to replicate in vertebrate cells [5]. Because of the host-restriction there is no vertebrate amplifying host that can maintain a successful viral lifecycle between the mosquito and vertebrate animal, which is the case for most arboviruses [6]. Therefore, the main mechanism of transmission and maintenance of ISVs is thought to be vertical transmission, in which the virus is passed transovarially from infected female mosquitoes to their offspring. This is supported by both laboratory and field studies, where offspring from ISV-infected female mosquitoes have tested positive for the correlating virus [3,7-11]. The mechanisms for how ISVs manage to establish an infection in the mosquito is, however, not known. In the case of arbovirus interaction and infection in the mosquito more research has been done. For an arbovirus to successfully transmit to a new host via a blood meal, it needs to enter and replicate in the salivary gland of the mosquito. However, there are barriers 
and tissue-specific antiviral mechanisms that the virus has to overcome for this to occur (Fig. 1). The first barrier is the midgut epithelial cells. The virus needs to enter and escape these cells before spreading to the haemolymph from where it can spread systemically to the rest of the body including the salivary glands via the haemolymph circulation [12-14]. Each of these barriers have tissue specific antiviral immune responses including the Toll, immune deficiency

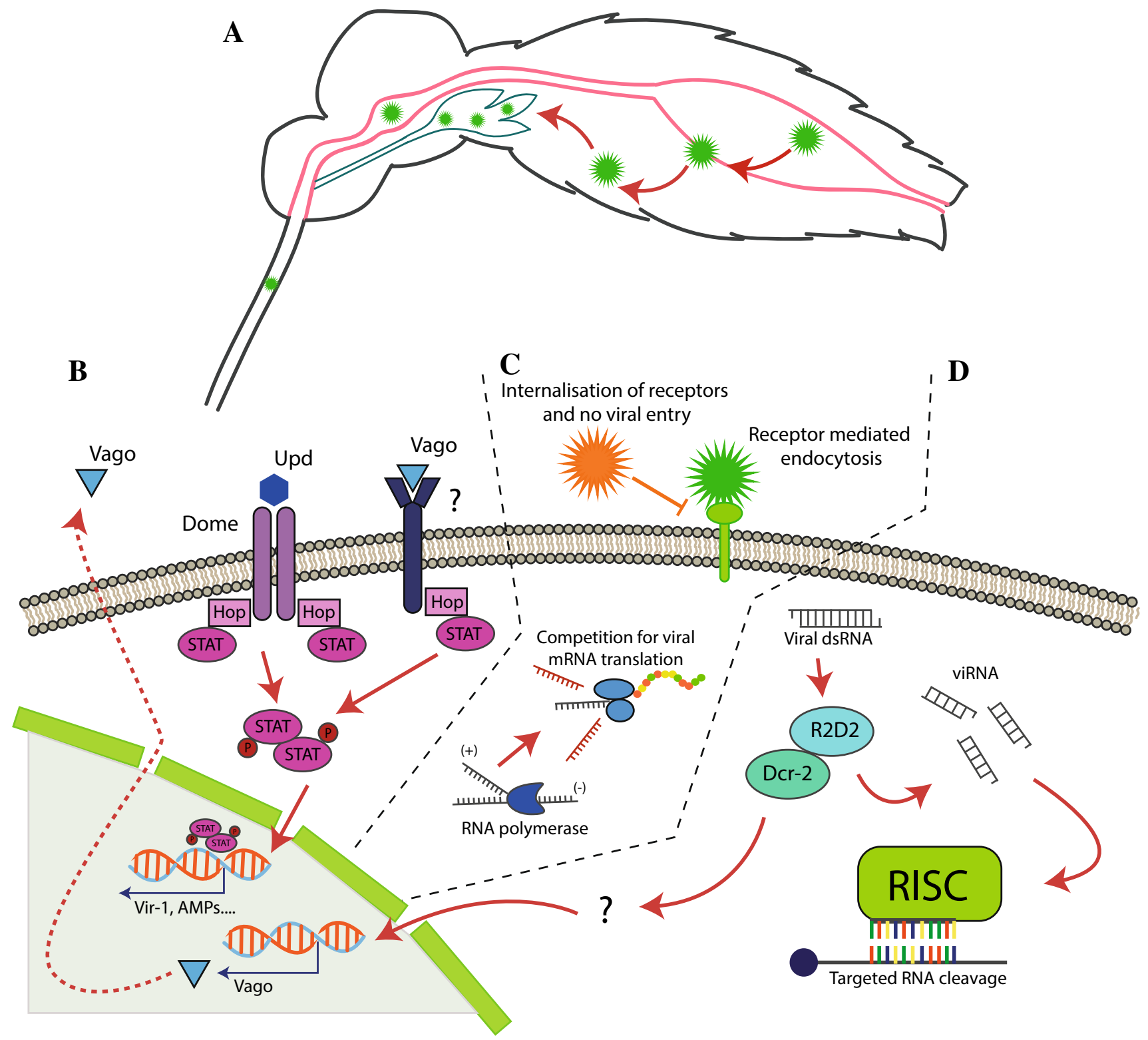

Fig. 1 Schematic overview of some of the mosquito antiviral mechanisms. a The mosquito ingests an arbovirus-infectious blood meal into the midgut. The virus enters and replicate in the midgut epithelial cells, after successful replication the virus escape into the haemolymph and spread systemically including to the salivary glands, where the virus enters and replicate before being transmitted via the saliva. b The JAK-STAT pathway is mainly activated when the transmembrane receptor Domeless (Dome) recognise extracellular unpaired ligands (Upd) leading to a conformational change that start autophosphorylation of Hop, which in turn phosphorylates Dome. This is leads to the phosphorylation and dimerization of STAT, resulting in a translocation of STAT dimers to the nucleus which activates the transcription of specific antiviral genes. c A primary viral infec- tion can block a secondary infection of a similar virus via mechanisms hypothesised to involve competition for, or modification of cellular resources reducing receptor binding, viral entry, RNA replication and translation of the secondary virus. d Viral dsRNA, either as replication intermediates or as part of the viral genomes, are processed by the Dcr-2-R2D2 complex to generate siRNAs of approximately $21-23$ bp of length. The siRNA are incorporated into the RNA-induced silencing complex (RISC) to recognize viral RNA for degradation. dsRNA can be sensed by the Dicer-2 DEcD/H-box helicase domain and via an unknown pathway activate expression and secretion of Vago, which can activate the JAK-STAT pathway via an unknown receptor in nearby cells 
factor (Imd), Janus kinase (JAK)- signalling transduction and activation of transcripts (STAT) pathways as well as RNA interference (RNAi) [15]. These pathways allow the mosquito to mount a defence against invading microorganisms, including viruses, and to understanding these antiviral mechanisms and control of viral infection in specific tissues is crucial and the backbone of novel control strategies that target arbovirus transmission in nature.

Of all arboviruses that cause human disease over $90 \%$ are vectored by mosquitoes [16] and with increasing temperatures, urbanization and global trade, the geographic range of different mosquitoes have expanded with associated increase in arbovirus disease burden [17-21]. Arboviruses are a global problem with, for example, annual outbreaks of dengue virus (DENV) in the Americas [22], yellow fever virus outbreaks in south America [23], West Nile virus (WNV) becoming endemic in Europe [24] and the emerging Zika virus (ZIKV) [25]. The global arbovirus disease burden and the lack of licensed drugs and vaccines, cause an urgent need of new tools for disease control. In recent years, researchers have begun to study vector competence, as evidence has suggested that the microbiome of the mosquito can alter the susceptibility of certain arboviruses. The most studied example is the endosymbiotic bacterium Wolbachia, which naturally infect a broad range of arthropods including many mosquito species and is maternally transmitted. Wolbachia has been proven to reduce the vector competence of important arboviruses in key mosquito species through various mechanisms [26]. In a similar manner, it is believed that ISVs could also have the potential to be utilised as biocontrol agents with proposing effects of superinfection exclusion, upregulation of the vector antiviral immune-response and maintenance in nature by transovarial transmission. In this review, we discuss research strengthening the hypothesis of ISVs being ancestral to arboviruses as well as provide a comprehensive description of ISVs effect on vector competence.

\section{Host-range restrictions of ISVs}

Insect-specific viruses are, as mentioned in the introduction, characterized by their incapacity to infect vertebrates. This can be assessed through, for example, viral inoculation of mammalian, avian, or amphibian cell lines [27-29] or by intracerebral infection of neonatal mice [29-31]. Host-range restrictions may exist at both the pre- and post-entry steps of the viral cycle. To be able to replicate the virus must enter the cell, deliver their genome, replicate and build new infectious virus particles. Each of these steps is an obstacle to be overcome by the virus [32]. To study the mechanisms behind host-range restrictions reverse genetic systems have proven to be a valuable tool through the ability of creating chimeric viruses $[28,31,33]$.
Insect-specific viruses are believed to be restricted by the innate immune system in the vertebrate cell, but there is evidence pointing to that this is not the only mechanism causing the restriction of host-range [34]. In contrast to arboviruses, who can replicate at temperatures up to $42{ }^{\circ} \mathrm{C}$ insect-specific viruses replicate only at ambient temperatures. In a study by Marklewitz et al. [35] replication of an insect-specific bunyavirus was decreased and even hindered by elevated temperatures in invertebrate cells where arboviral bunyaviruses were not. However, it is not sufficient to just lower the temperature to enable the replication of ISVs in mammalian cells [5, 29, 35].

The impact of the vertebrate innate immune system in host-restriction has been shown for the ISV Kamiti River virus (KRV). Knockdown of pattern recognition receptors (RIG-I, MDA5 and TLR3) led to an elevation of KRV vRNA levels in both Vero and human lung carcinoma cells (A549) which points to that several of these receptors are important in the detection and control of replication of KRV in vertebrate cells [34]. The study also showed that KRV was able to enter IRF3,5,7-/- mouse embryonic fibroblasts, translate and shed infectious particle, although at low levels, which suggests that the KRV is capable of replicating in vertebrate cells if the innate immunity pathways are silenced [34]. This is in contrast to the insect-specific alphavirus, Eilat virus (EILV), that is restricted at multiple levels. In a study from 2012 Nasar et al. showed that EILV is unable to replicate in vertebrate cells, even after genomic RNA was electroporated directly in the cytoplasm. It was hypothesized that this was due to incorrect interactions between the EILV RNA or its gene products with vertebrate cell cofactors. A study from 2015, using Sindbis virus and EILV chimeras, showed that EILV is actually blocked at two independent stages of the replication cycle, both at RNA replication step as well as at viral entry [5].

A study by Junglen et al. [33] also showed that the hostrange restriction can be at several levels of the viral life cycle. In this study chimera Yellow fever virus carrying envelope proteins from the insect-specific flavivirus Niénokoue virus (NIEV) was use to study host range restrictions. In the study, they observed that the first barrier against infection of vertebrate cells was viral entry. The YF/NIEV chimera grew, and produced new virus particles in C6/36 cells after infection, however, no virus was shown after infection of BHK cells, indication that NIEV was unable to mediate viral entry into vertebrate cells. Electroporation of RNA from the YF/NIEV clone showed growth and assembly of infectious particles in C6/36 but no replication nor assembly was seen in BHK cells indicating that NIEV is restricted at both attachment/entry as well as at the assembly/release level [33]. 


\section{Insect-specific virus evolution}

ISVs and arboviruses both infect and replicate in insect vectors and show evolutionary relationship. This fact suggests that arboviruses could have been ISVs that through evolution acquired the ability to expand their host-range to also include vertebrates. As mentioned in the introduction, almost all arboviruses and insect-specific viruses belong to the RNA virus order Bunyavirales (negative (-) sense ssRNA), or to the viral families Flaviviridae (postitive (+) sense, ssRNA), Reoviridae (dsRNA), Rhabodoviridae (-ssRNA) and Togaviridae (+ssRNA). RNA viruses lack the same proofreading mechanism as DNA viruses which results in greater plasticity and higher mutation rates [36]. Together with insect's affinity to live in large dense populations this could be one of the explanations to the great diversity of arbovirus hosts. Adaptations that provide a virus with the possibility to infect new hosts gives the virus the possibility to spread geographically. Altogether, this suggests that ISVs are a potential source of new arboviruses.

The idea that arboviruses originated from viruses found in arthropods is far from new, as it was first suggested more than 50 years ago [4]. Abundance of viral RNA in the arthropod transcriptome and high incidence of endogenous copies in the genome of arthropods suggests that ISVs have probably played a role in the evolution of RNA viruses [37]. Together with the fact that many insect-specific viruses appear to be vertically transmitted, this points to the fact that these viruses coexisted with their insect host for a long period of time [35, 38-41]. Reconstruction of phylogenetic ancestral hosts helps in the identification of ancestral host switching processes. It can also help predict in which direction the virus will spread to new hosts [42].

\section{Bunyavirales}

Arthropod hosts have been constructed for all deep tree nodes of the bunyavirus tree [35], which suggest that arboviruses from this order evolved from insect-specific viruses. It has also been suggested that an ancient insectborne bunyavirus lineage made the jump from insects to mammals and that these viruses comprise the presentday genus Hantavirus [43]. Ballinger et al. [38] shows evidence for that the insect-specific phasmaviruses are members of an ancient bunyavirus lineage grouping with Hantavirus, Orthobunyavirus, and Tospovirus. Bunyaviruses is a large and diverse genus composed of more than 350 viruses. Many of the bunyaviruses are pathogenic to human and animals but the genus also comprises several insect-specific viruses. These viruses are often divergent from arthropod viruses. Recent studies have discovered a number of insect-specific bunyaviruses, and furthermore some studies have also found nucleoproteins similar to bunyaviruses in the genome of non-blood feeding insects such as Drosophila. This finding suggests that there have been interactions between bunyaviruses and arthropods for over 20 million years [27, 37, 38, 44-48]. In a study by Marklewitz et al. [35] a reconstruction of an ancestral host for Bunyavirales was made. The results in the study points to that vertebrate, or dual-host tropism, have evolved several times during the evolution of viruses. For the Hantavirus, spread primarily by rodents, arthropod tropism is thought to have been lost in favour of vertebrate single tropism [35].

\section{Flaviviridae}

One of the first discovered insect-specific clades of flaviviruses, that includes cell fusing agent virus, Kamiti River virus and Culex flavivirus, branches at the base of the Flavivirus genus suggesting an arthropod virus origin of the arboviruses within the genus $[4,49,50]$. The same could be suggested for all flaviviruses as 12 flavivirus-like viruses of deep rooting lineages were identified in a range of arthropod species [51]. A second clade of insect-specific flaviviruses that cluster with the mosquito-borne flaviviruses have been found, although it is unclear at this point whether they lost dual-host tropism or maintained single host tropism from the root of the genus [4, 29, 50, 52].

\section{Togaviridae}

Two insect-specific viruses identified within the Alphavirus genus (Togaviridae): Eilat virus and Taï Forest alphavirus are both related basally to Western equine encephalitis virus which could suggest an insect-specific ancestor to Western equine encephalitis virus $[28,53]$. Yet, the rarity of insectspecific alphaviruses makes conclusions concerning the evolution in this genus hard to draw.

\section{Rhabdoviridae}

There are several insect-specific lineages in a number of places in the Rhabdoviridae family phylogenetic tree [54]. However, the existence of the bat- and human pathogenic lyssaviruses and the novirhabdoviruses, known to infect aquatic hosts, that are both situated in basal phylogenetic positions, could coincide with the idea of an insect-specific ancestor in the Rhabdoviridae family [32].

Insights in the cellular and genetic requirements for adaptation of insect-specific viruses to vertebrate hosts are of great importance. This information can potentially be 
used to predict potential future spill over of new pathogenic viruses from insects to humans and animals. Furthermore, there is great value in the knowledge of the origin and evolution of viruses, as it provides insight into the history of virus emergence and how viruses acquire the ability to infect new hosts and become more pathogenic.

\section{Vector competence studies}

Today mosquitoes are the subject of a wide variety of control strategies utilizing everything from bacteria, aquatic animals and chemicals [55-57]. Most of these strategies focus on reducing the vector abundance, often by targeting and killing mosquito larvae and/or adults. These strategies reduce or contain arbovirus transmission, but are cumbersome and costly. Mainly because of the dependence on community participation to access hidden breeding sites such as yards or gardens and the constant need to repeat the treatments and monitor levels of larvae and/or adults [58, 59]. To measure how effectively a hematophagous arthropod vector can transmit a virus (vectorial capacity) we need to consider several factors such as vector competence (the proportion of vectors that acquire an arbovirus infection and transmit it to a vertebrate), vector abundance, host specificity, vector longevity, the extrinsic incubation period and blood feeding frequency. All these factors have an impact on vectorial capacity and arbovirus disease burden and can be calculated with the equation

$C=\left(\mathrm{ma}^{2}\right)(\mathrm{pn})(b) /-\log (p)$,

where $C$ is the vectorial capacity, $b$ is the vector competence, $p$ is the daily probability of vector survival, $n$ is the extrinsic incubation period and $\mathrm{ma}^{2}$ is a combined value of blood feeding frequency with human biting rate $[60,61]$.

In recent years, researchers have looked at other options such as genetically modified vectors (GMVs) [62] and manipulation of the microbiome to enhance the antiviral resistance [63]. These strategies are focusing on the vector competence of the mosquito to carry and transmit arboviruses of human and animal importance. One advantage of these novel control strategies is the maintenance in the mosquito population, were both the GMVs and manipulation of the microbiome are vertically transmitted by female mosquitoes to their offspring $[7,64,65]$ and thus sustaining the transmission blocking effect. Manipulation of the microbiome can be done by altering the flora of bacteria, fungi or virus in the mosquito by, for example, introducing a microorganism with a known interfering effect. The most studied example is the use of the endosymbiotic bacterium named Wolbachia, which has repeatedly proven the ability to hinder infection of important arboviruses such as chikungunya virus (CHIKV), DENV, ZIKV and WNV [26, 66-68].
Moreover, the microbiome of the mosquito and specifically the midgut microbiome has shown to contribute to antiviral protection through several mechanisms. It serves as a physical barrier by blocking gut epithelial cells from pathogenic exposure [69], the microorganisms can produce secondary metabolites that inhibit arboviruses [70] and it also provides a basal immune activation, aggravating viral entry and replication [71, 72]. In this review, we will focus on the potential use of ISVs as biocontrol agents (Table 1) in a similar manner as Wolbachia. The relative genetic similarity between ISVs and arboviruses have a potential for replicative interference not only through upregulation of antiviral immuneresponses in the vector but also via a phenomenon called superinfection exclusion (Fig. 1c). Superinfection exclusion (or homologous interference) is a phenomenon where a primary viral infection can block a secondary infection of a similar virus. The molecular mechanisms of superinfection exclusion are hypothesised to involve competition for, or modification of cellular resources reducing receptor binding, viral entry, RNA replication and translation of the secondary virus [73]. Superinfection exclusion has, for example, been demonstrated for two different strain of WNV in Culex pipiens mosquitoes [74], WNV and St. Louis encephalitis virus (SLEV) in Culex quinquefasciatus [75] and in many different cell culture superinfection experiments [76-78].

\section{Culex flavivirus}

The ISV Culex flavivirus (CxFV) was first isolated in Japan in 2007 [79] and has since been isolated in Culex mosquitoes all over the globe [80-88]. Kent et al. was the first to evaluate the potential effect of $\mathrm{CxFV}$ to block propagation and transmission of arbovirus. In that study, prior infection of CxFV Izabal was evaluated for its effect on vector competence of WNV in Culex quinquefasciatus. They performed in vitro and in vivo experiments using Aedes albopictus C6/36 cells, two strains of Culex quinquefasciatus (Sebring and Honduras), CxFV Izabal and West Nile virus isolate GU-06-2256. Replication of WNV was monitored between day 0 and 14 in $\mathrm{CxFV}$-positive and CxFV-negative C6/36 cell and no significant inhibition of WNV growth was observed. In the in vivo experiments the two strains of Culex quinquefasciatus mosquitoes were intrathoracic inoculated with either $\mathrm{CxFV}$, heat-inactivated $\mathrm{CxFV}$ or a mock infection, all groups were exposed to a WNV-infectious blood meal of multiple titres 7 days post inoculation (dpi). 2 weeks following WNV exposure, individual mosquito bodies, legs and saliva were measured for WNV growth by plaque titration on Vero cells representing infection, dissemination and transmission. Similarly, to the in vitro experiment, there were no difference in WNV growth or transmission, and they conclude that CxFV Izabal have no effect on vector competence for WNV in Culex quinquefasciatus [89]. However, in 
Table 1 Summary of the papers discussed in the "Vector competence studies" subsection regarding the effect of different ISVs on vector competence

\begin{tabular}{|c|c|c|c|c|c|c|c|}
\hline Study & ISV & In vitro & In vivo & Control virus & Effect & Year & References \\
\hline Kent et al. & CxFV & Yes & Yes & WNV & No effect on vector competence for WNV & 2010 & [89] \\
\hline Bolling et al. & $\mathrm{CxFV}$ & Yes & Yes & WNV & $\begin{array}{l}\text { Early interference of infection, no effect on } \\
\text { transmission }\end{array}$ & 2012 & [65] \\
\hline Hopson-Peter et al. & PCV & Yes & No & WNV, MVEV & 10-43-fold growth inhibition in $\mathrm{C} 6 / 36$ cells & 2013 & [97] \\
\hline Kenney et al. & NHUV & Yes & No & WNV, SLEV, JEV & $\begin{array}{l}\text { 1.5 million-fold reduction for WNV, } \\
80 \text {-fold reduction for JEV and } 15,000 \text { - } \\
\text { fold reduction for SLEV in C6/36 }\end{array}$ & 2014 & [99] \\
\hline Goenaga et al. & NHUV & Yes & Yes & WNV & $\begin{array}{l}\text { 4000-fold reduction for WNV in vitro. No } \\
\text { significant effect in co-infected Cx. pipi- } \\
\text { ens. However, Co-infected Cx. quinque- } \\
\text { faciatus had a significant difference in } \\
\text { transmission at } 7 \text { and } 9 \mathrm{dpi}\end{array}$ & 2015 & {$[101]$} \\
\hline Kuwata et al. & $\mathrm{CxFV}$ & Yes & No & JEV, DENV & $\begin{array}{l}\text { Pre-existing CxFV infection do not sup- } \\
\text { press growth of a superinfecting flavivi- } \\
\text { rus but rather enhance virus release from } \\
\text { the NIID-CTR cells }\end{array}$ & 2015 & [93] \\
\hline Nasar et al. & EILV & Yes & Yes & $\begin{array}{l}\text { SINV, VEEV, EEEV, } \\
\text { WEEV, CHIKV }\end{array}$ & $\begin{array}{l}\text { In vitro results showed that EILV infection } \\
\text { reduced superinfecting virus production } \\
\text { by } 10 \text { to } 10,000 \text {-fold and delayed replica- } \\
\text { tion kinetics at least } 12-48 \text { h regardless } \\
\text { of virus or MOI. In vivo studies showed } \\
\text { a delay of dissemination from the midgut } \\
\text { by } 3 \text { days }\end{array}$ & 2015 & [31] \\
\hline Hall-Mendelin et al. & PCV & No & Yes & WNV & $\begin{array}{l}\text { Significantly lower infection rate in PCV- } \\
\text { infected } C x \text {. Annulirostris orally exposed } \\
\text { to WNV }\end{array}$ & 2016 & [98] \\
\hline Talavera et al. & $\mathrm{CxFV}$ & No & Yes & RFV & No effect & 2018 & [94] \\
\hline Schultz et al. & CFAV, PCLV & Yes & No & ZIKV, DENV \& LACV & $\begin{array}{l}90 \% \text { reduction of ZIKV \& DENV growth. } \\
\text { Complete inhibition of LACV at MOI } 0.1\end{array}$ & 2018 & [104] \\
\hline
\end{tabular}

the paper they discuss the limitation of infecting the mosquitoes by intrathoracic inoculation and that naturally infected mosquitoes would be preferable due to earlier studies [90, 91] showing that the route of infection affect the outcome of arbovirus superinfection [89].

In regard to this, Bolling et al. performed a similar study, using a laboratory colony of Culex pipiens naturally infected with $\mathrm{CxFV}$, to study transmission dynamics of $\mathrm{CxFV}$ as well as the effects of $\mathrm{CxFV}$ infection on vector competence of WNV. They first performed an in vitro experiment where CxFV infected C6/36 cells were challenged with WNV, the results showed that WNV growth curves in co-infected cells were significantly lower than WNV only infected cells between 84 and $156 \mathrm{~h}$ post infection, by $168 \mathrm{~h}$ post infection no difference could be observed. They followed up with an in vivo experiment using two Culex pipiens laboratory colonies, the CPCO (Culex pipiens-Colorado, CxFV-positive) and the CPIA (Culex pipiens-Iowa, CxFV-negative). Female mosquitoes from the two colonies were given a WNV infectious blood meal and infection, dissemination and transmission rates were compared at 7 and $14 \mathrm{dpi}$. Results after $7 \mathrm{dpi}$ showed that dissemination rate was significantly lower in the CxFV-positive mosquitoes and the infection rate was also lower but not significantly. However, there was no difference in the estimated transmission rate, and after $14 \mathrm{dpi}$ there was no statistically significant difference between the two colonies. Both the in vitro and in vivo experiments show an early time-point difference that may represent interference between $\mathrm{CxFV}$ and WNV, however, no difference in the transmission rates between $\mathrm{CxFV}$-positive and $\mathrm{CxFV}$ negative mosquitoes question the overall impact of $\mathrm{CxFV}$ on vector competence of WNV [65]. These two studies utilized the $\mathrm{C} 6 / 36$ cell lines in their in vitro experiments, this cell line is derived from Aedes albopictus mosquitoes, although WNV and CxFV are generally associated with Culex mosquitoes. Kuwata et al. studied the effect of CxFV in a Culex cell line derived from Culex tritaeniorhynchus embryos (NIID-CTR) [92]. They generated a NIID-CTR cell line persistently infected with $\mathrm{CxFV}$, which showed no CPE and grew normally as compared to the original NIID-CTR. The CxFV-positive and the original cell lines were challenged with Japanese encephalitis virus (JEV) or DENV, cell growth and DENV/JEV replication were analysed every $24 \mathrm{~h}$ between day 1 and $7 \mathrm{dpi}$. Superinfection 
with JEV represented an arbovirus generally associated with Culex mosquitoes and DENV which is unrelated to Culex mosquitoes served as a control to compare the results of the JEV-CxFV system. Experimental results showed that CxFV-positive cells significantly enhanced replication and/ or release of JEV at 4 dpi compared to the JEV-only infected cells. The increase of the JEV-titre appeared at the same time as a $25 \%$ decrease in cell number of the CxFV-positive cells, which may explain the massive increase by a release of JEV particles into the medium. The JEV-titre stayed significantly higher compared to the control throughout the experiment. CxFV-positive cells challenged with DENV also showed a significant higher titre after $4 \mathrm{dpi}$, however, cells showed no $\mathrm{CPE}$ and there was no reduction in cell number. From this they conclude that pre-existing CxFV infection do not suppress growth of a superinfecting flavivirus but rather enhance virus release from the NIID-CTR cells [93].

A study by Talavera et al. investigated the ability of Rift Valley fever virus (RVFV) to infect, disseminate and be transmitted by Culex pipiens pre-infected with CxFV. To evaluate if $\mathrm{CxFV}$ can interfere with a viruses from different genera, $\mathrm{CxFV}$ (Flavivirus) and RVFV (phlebovirus). Female Culex pipiens were intrathoracically infected with $\mathrm{CxFV}$ and then received a RVFV-infectious blood meal, 14 days post RVFV exposure the mosquitoes were dissected and analysed for infection, dissemination and transmission. Results showed no significant difference in any of the parameter analysed compared to the control and they conclude that CxFV have no effect on RVFV replication in Culex pipiens mosquitoes [94].

To draw any conclusion on the effect of CxFV is difficult, most studies are inconclusive or have conflicting result, e.g. Newman et al. saw a strong correlation between WNV and $\mathrm{CxFV}$ infection rates, where field caught mosquitoes in Chicago had a four-fold increased likelihood of CxFV infection of WNV positive mosquito pools, compared to WNVnegative pools [95]. While, Crockett et al. saw no association between $\mathrm{WNV}$ and $\mathrm{CxFV}$ infection rates in mosquitoes collected in south-eastern united states [96].

\section{Palm Creek virus}

Hobson-Peters et al. isolated an ISV from Coquillettidia xanthogaster mosquitoes in northern Australia and the virus was named Palm Creek virus (PCV) after its place of isolation. In the same paper, they showed that the use of PCV pre-infected C6/36 cells resulted in suppressed replication of both WNV and Murray Valley encephalitis virus (MVEV) by 10-43 folds compared to WNV or MVEV-only infected C6/36 cells [97].

Hall-Mendelin et al. did a follow up study and performed in vivo experiments studying vertical transmission, modes of transmission and WNV replication interference of PCV.
They used relevant arbovirus vectors of Australia and focused on Culex annulirostris, which is an important vector of WNV and MVEV in that region. In the transmission experiments, no Culex annulirostris were infected by PCV when orally exposed via a PCV-infectious blood meal, and no progeny reared from $\mathrm{PCV}$-infected Culex annulirostris were PCV positive, showing that PCV is probably host-specific to Coquillettidia xanthogaster. However, Culex annulirostris can support PCV infection and $100 \%$ were infected when intrathoracic inoculated. Interestingly, experimental results showed that PCV positive Culex annulirostris had a significantly lower WNV infection rate than PCV negative when given a WNV-infectious blood meal. To understand the PCV-mediated exclusion of WNV via the oral infection route they examined the tissue tropism of PCV with immunohistochemistry and found that the virus is specifically localized in the epithelial lining of the midgut and was not present in any other tissues [98]. The exact mechanism of PCV blocking of WNV is not revealed, we can, however, speculate that it is a competition of cellular resources between PCV and WNV [73], or that PCV upregulate the immune activation hindering establishment of WNV entry and replication [71, 72].

\section{Nhumirim virus}

Another ISV that has been evaluated for its capacity to supress replication of important arboviruses is Nhumirim virus (NHUV) that was isolated in the Pantanal region of Brazil [99, 100]. Kenney et al. preformed co-infection experiments in C6/36 cell with prior or concurrent infection of NHUV and challenged with either WNV, SLEV or JEV. NHUV showed to be a very potent inhibitor of all of these viruses with peak-titre differences translated to 1.5 million-fold reduction of $\mathrm{WNV}, 80$-fold reduction of JEV and a 15,000-fold reduction for SLEV compared to the arbovirus only infected controls. A study done by Goenaga et al. further support the inhibition effect in vitro. Experiments, performed with C6/36 cells and the Aedes albopictus cell line C7/10, showed a 4000-fold decrease in WNV growth in both cell-lines pre-infected with NHUV. However, when Culex pipiens were coinoculated intrathoracically with NHUV and WNV in a 10:1 ratio and processed at 14 dpi no significant difference in infection, dissemination and transmission was seen compared to the control. They further assessed the capacity of Culex quinquefasciatus, co-inoculated with NHUV and WNV, to become infected and transmit WNV. Mosquitoes were co-inoculated and harvested at 3, 5, 7 and $9 \mathrm{dpi}$, the results demonstrated $100 \% \mathrm{WNV}$ infection at all time points, except at 3 dpi where a $91 \%$ infection rate was observed, this was significantly lower than the control. Although there was no significant difference 
in WNV titres in bodies and saliva they saw a trend of lower WNV titres in the saliva of co-infected mosquitoes and there was a significant difference in the transmission rate of mosquitoes that were co-infecting at 7 and $9 \mathrm{dpi}$ compared to the control [101]. NHUV showed promising in vitro interference of superinfecting viruses, however, the in vivo experiment was not clear. Co-inoculated Culex quinquefasciatus showed a significantly lower transmission rate at the later time points compared to the control, which could indicate an interference of WNV replication in the salivary gland. This was however, not observed in the Culex pipiens mosquitoes, indicating that NHUV only have this effect in some Culex species. In the paper, they discuss that further studies with prior infection of NHUV instead of co-infection could increase the interfering effect and would also simulate a more natural infection [101].

\section{Eilat virus}

Eilat virus (EILV) is an insect-specific alphavirus that was isolated from a pool of Anopheles coustani mosquitoes caught in the Negev desert of Israel [28] and have been used as a backbone in a vaccine platform [102]. Nasar et al. investigated the ability of EILV to interfere with superinfecting alphaviruses such as Sindbis virus (SINV), chikungunya virus (CHIKV) and western (WEEV), eastern (EEEV) and Venezuelan equine encephalitis virus (VEEV) in C7/10 cells and in Aedes aegypti mosquitoes. To investigate heterologous interference, $\mathrm{C} 7 / 10$ cells were infected with EILV at a MOI of 10 or a mock infection, $24 \mathrm{~h}$ post the initial infection cells were superinfected with either SINV, VEEV, EEEV, WEEV or CHIKV at a MOI of 1 or 0.1 . In vitro results showed that EILV infection reduced the superinfecting virus production by 10 to 10,000 -fold and delayed replication kinetics at least $12-48 \mathrm{~h}$ regardless of virus or MOI. To investigate if similar results are achieved in vivo, Aedes aegypti mosquitoes were infected with EILV by intrathoracic inoculation and 7 days post inoculation mosquitoes were provided a blood meals containing CHIKV of $10^{5} \mathrm{PFU} / \mathrm{ml}$. Infection rate and CHIKV dissemination were analysed and results showed that EILV infection delayed dissemination from the midgut by 3 days, however, after 5 and 7 days post superinfection the dissemination rates were higher or identical as compared to the mock infected group. In the paper, they discuss several interesting factors regarding why the EILV-mediated interference was not observed beyond 5 days post superinfection in mosquitoes. For example, that EILV replication may decrease over time which reduce the interfering capacity, or that EILV and CHIKV may have different cell tropisms and are therefore not competing for the cellular resources [31].

\section{Dual ISV infection}

Lastly, a study done by Schultz et al. evaluated the effect of dual insect-specific virus infection on replication of ZIKV, DENV-2 and La Crosse virus (LACV) in the Aedes albopictus derived cell line Aa23. Their cell-line was persistently infected with CFAV and was further inoculated with the Phasi Charoen like virus (PCLV) [103] generating a CFAV-PCLV positive Aa23 cell line. These and the Aa23 control cells (CFAV-only) were challenged with ZIKV, DENV and LACV at a low MOI of 0.1 and a high MOI of 10, respectively, and arbovirus growth was analysed after 3 and $6 \mathrm{dpi}$. They observed a $90 \%$ reduction of ZIKV growth at both the low and high MOI which was significantly lower than the control. DENV-2 had a similar reduction (90\%) of growth at the low MOI, however, in the case of the high MOI no difference in growth compared to the control was observed. LACV had a complete inhibition at the low MOI and no virus growth was detectable in the CFAV-PCLV positive cells, the high MOI resulted in a $90-99.9 \%$ reduction of LACV growth. Dual-ISV infection of the two virus families Flaviviridae (CFAV) and a Peribunyaviradae (PCLV) showed a robust interference and in one case a complete blockage of arboviruses within the same genus [104].

The majority of all vector competence studies, done so far have used the Aedes albopictus C6/36 cell line (Table 1), this cell line has a defective RNAi response [105] which question the biological relevance from these experiments, due to the central role the RNAi plays in controlling arbovirus infection in the vector. The C6/36 cell line is very useful and easy to work with, but additional immune-competent mosquito cell lines such as the Aa23 [106], Aag2 [107] or HSU [108] should be included in studies regarding vector competence. Further, recent studies have showed that laboratory mosquito cell lines can be persistently infected with ISVs, without any CPE or affecting the cell growth. Schultz et al. screened their mosquito cell lines and discovered a persistent infection of CFAV and PCLV [104]. Further, Weger-Lucarelli et al. screened the four widely used mosquito cell lines C6/36, U4.4, Aag2 and HSU and confirmed that all four cell lines were persistently infected with several viruses [109]. This should to be considered when designing an experiment or analysing data generated from these cell lines.

Novel biocontrol strategies should aim to be inexpensive, effective, environmentally friendly, safe and self-sustaining. The use of ISVs as a tool for control of arbovirus transmission have the potential to fulfil these requirements, however, ISV research is still in an early stage and further research is needed before ISVs are implemented in real life settings. 


\section{Concluding remarks}

Today's technologies have enabled a new era of viral discoveries, where large metagenomics studies of environmental samples or animals are both possible and affordable. Insect-specific viruses are a relative new group of viruses with many interesting properties that have the potential to be utilized to further understand the evolution of viruses and to possibly aid the prevention of arbovirus transmission.

Acknowledgements This work has been supported by the Swedish University of Agricultural Sciences (SLU) vice chancellor career support as well as by the Swedish Research Council VR (2016-01251).

\section{Compliance with ethical standards}

Conflict of interest The authors have no conflict of interest to declare.

Research involving human participants and/or animals This article does not contain any studies with human participants or animals performed by any of the authors.

Open Access This article is distributed under the terms of the Creative Commons Attribution 4.0 International License (http://creativeco mmons.org/licenses/by/4.0/), which permits unrestricted use, distribution, and reproduction in any medium, provided you give appropriate credit to the original author(s) and the source, provide a link to the Creative Commons license, and indicate if changes were made.

\section{References}

1. Stollar V, Thomas VL (1975) An agent in the Aedes aegypti cell line (Peleg) which causes fusion of Aedes albopictus cells. Virology 64(2):367-377

2. Cammisa-Parks H et al (1992) The complete nucleotide sequence of cell fusing agent (CFA): homology between the nonstructural proteins encoded by CFA and the nonstructural proteins encoded by arthropod-borne flaviviruses. Virology 189(2):511-524

3. Cook $\mathrm{S}$ et al (2006) Isolation of a new strain of the flavivirus cell fusing agent virus in a natural mosquito population from Puerto Rico. J Gen Virol 87(Pt 4):735-748

4. Bolling BG et al (2015) Insect-specific virus discovery: significance for the arbovirus community. Viruses 7(9):4911-4928

5. Nasar F et al (2015) Eilat virus host range restriction is present at multiple levels of the virus life cycle. J Virol 89(2):1404-1418

6. Weaver SC, Barrett AD (2004) Transmission cycles, host range, evolution and emergence of arboviral disease. Nat Rev Microbiol 2(10):789-801

7. Saiyasombat R et al (2011) Evidence of efficient transovarial transmission of Culex flavivirus by Culex pipiens (Diptera: Culicidae). J Med Entomol 48(5):1031-1038

8. Bolling BG et al (2011) Insect-specific flaviviruses from Culex mosquitoes in Colorado, with evidence of vertical transmission. Am J Trop Med Hyg 85(1):169-177

9. Auguste AJ et al (2015) A newly isolated reovirus has the simplest genomic and structural organization of any reovirus. J Virol 89(1):676-687
10. Haddow AD et al (2013) First isolation of Aedes flavivirus in the Western Hemisphere and evidence of vertical transmission in the mosquito Aedes (Stegomyia) albopictus (Diptera: Culicidae). Virology 440(2):134-139

11. Sang RC et al (2003) Isolation of a new flavivirus related to cell fusing agent virus (CFAV) from field-collected flood-water Aedes mosquitoes sampled from a dambo in central Kenya. Arch Virol 148(6):1085-1093

12. Girard YA, Klingler KA, Higgs S (2004) West Nile virus dissemination and tissue tropisms in orally infected Culex pipiens quinquefasciatus. Vector Borne Zoonotic Dis 4(2):109-122

13. Romoser WS et al (2004) Evidence for arbovirus dissemination conduits from the mosquito (Diptera: Culicidae) midgut. J Med Entomol 41(3):467-475

14. Salazar MI et al (2007) Dengue virus type 2: replication and tropisms in orally infected Aedes aegypti mosquitoes. BMC Microbiol 7:9

15. Cheng $G$ et al (2016) Mosquito Defense Strategies against Viral Infection. Trends Parasitol 32(3):177-186

16. McGraw EA, O’Neill SL (2013) Beyond insecticides: new thinking on an ancient problem. Nat Rev Microbiol 11(3):181-193

17. Sutherst RW (2004) Global change and human vulnerability to vector-borne diseases. Clin Microbiol Rev 17(1):136-173

18. Weaver SC, Reisen WK (2010) Present and future arboviral threats. Antiviral Res 85(2):328-345

19. Weaver SC (2013) Urbanization and geographic expansion of zoonotic arboviral diseases: mechanisms and potential strategies for prevention. Trends Microbiol 21(8):360-363

20. Soverow JE et al (2009) Infectious disease in a warming world: how weather influenced West Nile virus in the United States (2001-2005). Environ Health Perspect 117(7):1049-1052

21. Cleton $\mathrm{N}$ et al (2012) Come fly with me: review of clinically important arboviruses for global travelers. J Clin Virol 55(3):191-203

22. Messina JP et al (2014) Global spread of dengue virus types: mapping the 70 year history. Trends Microbiol 22(3):138-146

23. Romano AP et al (2014) Yellow Fever outbreaks in unvaccinated populations, Brazil, 2008-2009. PLoS Negl Trop Dis 8(3): 2740

24. Rizzoli A et al (2015) The challenge of West Nile virus in Europe: knowledge gaps and research priorities. Euro Surveill. 20(20):21135

25. Fauci AS, Morens DM (2016) Zika virus in the Americas-yet another arbovirus threat. N Engl J Med 374(7):601-604

26. Blagrove MS et al (2012) Wolbachia strain wMel induces cytoplasmic incompatibility and blocks dengue transmission in Aedes albopictus. Proc Natl Acad Sci USA 109(1):255-260

27. Marklewitz M et al (2013) Discovery of a unique novel clade of mosquito-associated bunyaviruses. J Virol 87(23):12850-12865

28. Nasar F et al (2012) Eilat virus, a unique alphavirus with host range restricted to insects by RNA replication. Proc Natl Acad Sci USA 109(36):14622-14627

29. Huhtamo E et al (2014) Novel flaviviruses from mosquitoes: mosquito-specific evolutionary lineages within the phylogenetic group of mosquito-borne flaviviruses. Virology 464-465:320-329

30. Auguste AJ et al (2014) Characterization of a novel Negevirus and a novel Bunyavirus isolated from Culex (Culex) declarator mosquitoes in Trinidad. J Gen Virol 95(Pt 2):481-485

31. Nasar F et al (2015) Eilat virus induces both homologous and heterologous interference. Virology 484:51-58

32. Halbach R, Junglen S, van Rij RP (2017) Mosquito-specific and mosquito-borne viruses: evolution, infection, and host defense. Curr Opin Insect Sci 22:16-27 
33. Junglen $\mathrm{S}$ et al (2017) Host range restriction of insect-specific flaviviruses occurs at several levels of the viral life cycle. mSphere. 2(1): $\mathrm{e} 00375$

34. Tree MO et al (2016) Insect-specific flavivirus infection is restricted by innate immunity in the vertebrate host. Virology 497:81-91

35. Marklewitz M et al (2015) Evolutionary and phenotypic analysis of live virus isolates suggests arthropod origin of a pathogenic RNA virus family. Proc Natl Acad Sci USA 112(24):7536-7541

36. Domingo E, Parrish CR, Holland JJ, (eds) (2008) CHAPTER 16-arbovirus evolution. In: Origin and evolution of viruses (2nd edn.). Academic Press, London, pp 351-391

37. Li CX et al (2015) Unprecedented genomic diversity of RNA viruses in arthropods reveals the ancestry of negative-sense RNA viruses. Elife 4:e05378

38. Ballinger MJ et al (2014) Discovery and evolution of bunyavirids in arctic phantom midges and ancient bunyavirid-like sequences in insect genomes. J Virol 88(16):8783-8794

39. Dudas G, Obbard DJ Are arthropods at the heart of virus evolution?. Elife, 2015. 4:e06837

40. Fort $\mathrm{P}$ et al (2012) Fossil rhabdoviral sequences integrated into arthropod genomes: ontogeny, evolution, and potential functionality. Mol Biol Evol 29(1):381-390

41. Crochu $S$ et al (2004) Sequences of flavivirus-related RNA viruses persist in DNA form integrated in the genome of Aedes spp. mosquitoes. J Gen Virol 85(Pt 7):1971-1980

42. Drexler JF et al (2012) Bats host major mammalian paramyxoviruses. Nat Commun 3:796

43. Plyusnin A, Sironen T (2014) Evolution of hantaviruses: cospeciation with reservoir hosts for more than 100 MYR. Virus Res 187:22-26

44. Cook S et al (2013) Novel virus discovery and genome reconstruction from field RNA samples reveals highly divergent viruses in dipteran hosts. PLoS ONE 8(11):e80720

45. Marklewitz M et al (2011) Gouleako virus isolated from West African mosquitoes constitutes a proposed novel genus in the family Bunyaviridae. J Virol 85(17):9227-9234

46. Qin XC et al (2014) A tick-borne segmented RNA virus contains genome segments derived from unsegmented viral ancestors. Proc Natl Acad Sci USA 111(18):6744-6749

47. Tokarz R et al (2014) Virome analysis of Amblyomma americanum, Dermacentor variabilis, and Ixodes scapularis ticks reveals novel highly divergent vertebrate and invertebrate viruses. J Virol 88(19):11480-11492

48. Tokarz R et al (2014) Genome characterization of Long Island tick rhabdovirus, a new virus identified in Amblyomma americanum ticks. Virol J 11:26

49. Cook S et al (2012) Molecular evolution of the insect-specific flaviviruses. J Gen Virol 93(Pt 2):223-234

50. Blitvich BJ, Firth AE (2015) Insect-specific flaviviruses: a systematic review of their discovery, host range, mode of transmission, superinfection exclusion potential and genomic organization. Viruses 7(4):1927-1959

51. Shi M et al (2016) Divergent viruses discovered in arthropods and vertebrates revise the evolutionary history of the flaviviridae and related viruses. J Virol 90(2):659-669

52. Junglen S (2016) Evolutionary origin of pathogenic arthropodborne viruses-a case study in the family Bunyaviridae. Curr Opin Insect Sci 16:81-86

53. Hermanns K et al (2017) Discovery of a novel alphavirus related to Eilat virus. J Gen Virol 98(1):43-49

54. Contreras MA et al (2017) Almendravirus: a proposed new genus of rhabdoviruses isolated from mosquitoes in tropical regions of the Americas. Am J Trop Med Hyg 96(1):100-109

55. Anderson JF et al (2011) Control of mosquitoes in catch basins in Connecticut with Bacillus thuringiensis israelensis,
Bacillus sphaericus, and spinosad. J Am Mosq Control Assoc 27(1):45-55

56. Bonds JA (2012) Ultra-low-volume space sprays in mosquito control: a critical review. Med Vet Entomol 26(2):121-130

57. Walton WE (2007) Larvivorous fish including Gambusia. J Am Mosq Control Assoc 23(2 Suppl):184-220

58. Luz PM et al (2011) Dengue vector control strategies in an urban setting: an economic modelling assessment. Lancet 377(9778):1673-1680

59. WHO, Global Strategy for dengue prevention and control, 20122020. WHO report, 2012. WHO/HTM/NTD/VEM/2012.5: p. 43

60. Delatte $\mathrm{H}$ et al (2010) Blood-feeding behavior of Aedes albopictus, a vector of Chikungunya on La Reunion. Vector Borne Zoonotic Dis 10(3):249-258

61. Ciota AT, Kramer LD (2013) Vector-virus interactions and transmission dynamics of West Nile virus. Viruses 5(12):3021-3047

62. Terenius $\mathrm{O}$ et al (2008) Molecular genetic manipulation of vector mosquitoes. Cell Host Microbe 4(5):417-423

63. Jupatanakul N, Sim S, Dimopoulos G (2014) The insect microbiome modulates vector competence for arboviruses. Viruses 6(11):4294-4313

64. Lutomiah JJ et al (2007) Infection and vertical transmission of Kamiti river virus in laboratory bred Aedes aegypti mosquitoes. J Insect Sci 7:1-7

65. Bolling BG et al (2012) Transmission dynamics of an insectspecific flavivirus in a naturally infected Culex pipiens laboratory colony and effects of co-infection on vector competence for West Nile virus. Virology 427(2):90-97

66. Caragata EP, Dutra HL, Moreira LA (2016) Inhibition of Zika virus by Wolbachia in Aedes aegypti. Microb Cell 3(7):293-295

67. Dodson BL et al (2014) Wolbachia enhances West Nile virus (WNV) infection in the mosquito Culex tarsalis. PLoS Negl Trop Dis 8(7):e2965

68. Dutra HL et al (2016) Wolbachia blocks currently circulating Zika virus isolates in brazilian aedes aegypti mosquitoes. Cell Host Microbe 19(6):771-774

69. Cirimotich CM, Ramirez JL, Dimopoulos G (2011) Native microbiota shape insect vector competence for human pathogens. Cell Host Microbe 10(4):307-310

70. Joyce JD et al (2011) Interactions between La Crosse virus and bacteria isolated from the digestive tract of Aedes albopictus (Diptera: Culicidae). J Med Entomol 48(2):389-394

71. Xi Z, Ramirez JL, Dimopoulos G (2008) The Aedes aegypti toll pathway controls dengue virus infection. PLoS Pathog 4(7):e1000098

72. Ramirez JL et al (2012) Reciprocal tripartite interactions between the Aedes aegypti midgut microbiota, innate immune system and dengue virus influences vector competence. PLoS Negl Trop Dis 6(3):e1561

73. Lee YM et al (2005) Dual mechanisms of pestiviral superinfection exclusion at entry and RNA replication. J Virol 79(6):3231-3242

74. Rozeboom LE, Kassira EN (1969) Dual infections of mosquitoes with strains of West Nile virus. J Med Entomol 6(4):407-411

75. Pesko K, Mores CN (2009) Effect of sequential exposure on infection and dissemination rates for West Nile and St. Louis encephalitis viruses in Culex quinquefasciatus. Vector Borne Zoonotic Dis 9(3):281-286

76. Zebovitz E, Brown A (1968) Interference among group A arboviruses. J Virol 2(11):1283-1289

77. Karpf AR et al (1997) Superinfection exclusion of alphaviruses in three mosquito cell lines persistently infected with Sindbis virus. J Virol 71(9):7119-7123

78. Pepin KM, Lambeth K, Hanley KA (2008) Asymmetric competitive suppression between strains of dengue virus. BMC Microbiol 8:28 
79. Hoshino K et al (2007) Genetic characterization of a new insect flavivirus isolated from Culex pipiens mosquito in Japan. Virology 359(2):405-414

80. Farfan-Ale JA et al (2009) Detection of RNA from a novel West Nile-like virus and high prevalence of an insect-specific flavivirus in mosquitoes in the Yucatan Peninsula of Mexico. Am J Trop Med Hyg 80(1):85-95

81. Morales-Betoulle ME et al (2008) Culex flavivirus isolates from mosquitoes in Guatemala. J Med Entomol 45(6):1187-1190

82. Blitvich BJ et al (2009) Genomic sequence and phylogenetic analysis of Culex flavivirus, an insect-specific flavivirus, isolated from Culex pipiens (Diptera: Culicidae) in Iowa. J Med Entomol 46(4):934-941

83. Kyaw Kyaw A et al (2018) Isolation and genomic characterization of Culex flaviviruses from mosquitoes in Myanmar. Virus Res 247:120-124

84. Goenaga S et al (2014) New strains of Culex flavivirus isolated in Argentina. J Med Entomol 51(4):900-906

85. Kim DY et al (2009) Characterization of Culex Flavivirus (Flaviviridae) strains isolated from mosquitoes in the United States and Trinidad. Virology 386(1):154-159

86. Huanyu $\mathrm{W}$ et al (2012) Isolation and identification of a distinct strain of Culex Flavivirus from mosquitoes collected in Mainland China. Virol J 9:73

87. Roiz D et al (2009) Detection of novel insect flavivirus sequences integrated in Aedes albopictus (Diptera: Culicidae) in Northern Italy. Virol J 6:93

88. Vazquez A et al (2012) Novel flaviviruses detected in different species of mosquitoes in Spain. Vector Borne Zoonotic Dis 12(3):223-229

89. Kent RJ, Crabtree MB, Miller BR (2010) Transmission of West Nile virus by Culex quinquefasciatus say infected with Culex Flavivirus Izabal. PLoS Negl Trop Dis 4(5):e671

90. Borucki MK et al (1999) Bunyavirus superinfection and segment reassortment in transovarially infected mosquitoes. J Gen Virol 80 (Pt 12):3173-3179

91. Beaty BJ et al (1983) Interference between bunyaviruses in Aedes triseriatus mosquitoes. Virology 127(1):83-90

92. Kuwata R et al (2012) Establishment and characterization of a cell line from the mosquito Culex tritaeniorhynchus (Diptera: Culicidae). In Vitro Cell Dev Biol Anim 48(6):369-376

93. Kuwata R et al (2015) Analysis of Mosquito-Borne Flavivirus Superinfection in Culex tritaeniorhynchus (Diptera: Culicidae) Cells Persistently Infected with Culex Flavivirus (Flaviviridae). J Med Entomol 52(2):222-229

94. Talavera $\mathrm{S}$ et al (2018) Culex flavivirus infection in a Culex pipiens mosquito colony and its effects on vector competence for Rift Valley fever phlebovirus. Parasit Vectors 11(1):310

95. Newman CM et al (2011) Culex flavivirus and West Nile virus mosquito coinfection and positive ecological association in Chicago, United States. Vector Borne Zoonotic Dis 11(8):1099-1105
96. Crockett RK et al (2012) Culex flavivirus and West Nile virus in Culex quinquefasciatus populations in the southeastern United States. J Med Entomol 49(1):165-174

97. Hobson-Peters J et al (2013) A new insect-specific flavivirus from northern Australia suppresses replication of West Nile virus and Murray Valley encephalitis virus in co-infected mosquito cells. PLoS ONE 8(2):e56534

98. Hall-Mendelin S et al (2016) The insect-specific Palm Creek virus modulates West Nile virus infection in and transmission by Australian mosquitoes. Parasit Vectors 9(1):414

99. Kenney JL et al (2014) Characterization of a novel insect-specific flavivirus from Brazil: potential for inhibition of infection of arthropod cells with medically important flaviviruses. J Gen Virol 95(Pt 12):2796-2808

100. Pauvolid-Correa A et al (2015) Nhumirim virus, a novel flavivirus isolated from mosquitoes from the Pantanal, Brazil. Arch Virol 160(1):21-27

101. Goenaga $\mathrm{S}$ et al (2015) Potential for co-infection of a mosquitospecific flavivirus, Nhumirim virus, to block West Nile virus Transmission in Mosquitoes. Viruses 7(11):5801-5812

102. Erasmus JH et al (2018) Novel insect-specific eilat virus-based chimeric vaccine candidates provide durable, mono- and multivalent, single-dose protection against lethal alphavirus challenge. J Virol,. 92(4):e01274

103. Chandler JA et al (2014) Metagenomic shotgun sequencing of a Bunyavirus in wild-caught Aedes aegypti from Thailand informs the evolutionary and genomic history of the Phleboviruses Virology 464-465:312-319

104. Schultz MJ, Frydman HM, Connor JH (2018) Dual Insect specific virus infection limits Arbovirus replication in Aedes mosquito cells. Virology 518:406-413

105. Brackney DE et al (2010) C6/36 Aedes albopictus cells have a dysfunctional antiviral RNA interference response. PLoS Negl Trop Dis 4(10):e856

106. O'Neill SL et al (1997) In vitro cultivation of Wolbachia pipientis in an Aedes albopictus cell line. Insect Mol Biol 6(1):33-39

107. Lan Q, Fallon AM (1990) Small heat shock proteins distinguish between two mosquito species and confirm identity of their cell lines. Am J Trop Med Hyg 43(6):669-676

108. Hsu SH, Mao WH, Cross JH (1970) Establishment of a line of cells derived from ovarian tissue of Clex quinquefasciatus Say. J Med Entomol 7(6):703-707

109. Weger-Lucarelli $\mathrm{J}$ et al (2018) Adventitious viruses persistently infect three commonly used mosquito cell lines. Virology 521:175-180

Publisher's Note Springer Nature remains neutral with regard to jurisdictional claims in published maps and institutional affiliations. 